\title{
THE CROSSOVER AND MAGNETO-OPTICAL EFFECTS IN SUNSPOT SPECTRA
}

\author{
V. M. GRIGORYEV and J. M. KATZ \\ Siberian Institute of Terrestrial Magnetism, Ionosphere and \\ Radio Propagation, Irkutsk, U.S.S.R.
}

\begin{abstract}
Two peculiarities of the magnetic splitting of a line in sunspot spectra have been investigated. The one is that in a rather small region of the penumbra near the umbra-penumbra boundary the $\pi$-component is absent in one circular polarization spectrum while both $\sigma$-components are present. In the spectrum of opposite circular polarization the $\sigma$-components are absent but the $\pi$-component is present. The second peculiarity consists of the anomalous splitting of the $\pi$-component of Zeeman triplets which are of the same and opposite signs in comparison with splitting of the $\sigma$-components. The nature of these effects is discussed.
\end{abstract}

